\title{
Erratum to: Explosive Expansion of $\beta \gamma$-Crystallin Genes in the Ancestral Vertebrate
}

Guido Kappé • Andrew G. Purkiss •

Siebe T. van Genesen - Christine Slingsby •

Nicolette H. Lubsen

Published online: 15 September 2010

(C) Springer Science+Business Media, LLC 2010

Erratum to: J Mol Evol (2010) 71:219-230

DOI 10.1007/s00239-010-9379-2

Figure legends are erroneously switched around in the published article. The legend to Fig. 2 in the paper actually refers to Fig. 5, while the legend to Fig. 5 in the paper is the legend to Fig. 2.

Correct figures with the subsequent legends are given overleaf.

The online version of the original article can be found under doi:10.1007/s00239-010-9379-2.

G. Kappé · S. T. van Genesen · N. H. Lubsen $(\bowtie)$

Biomolecular Chemistry 271, NCMLS, Radboud University

Nijmegen, P.O. Box 9101, 6500 HB Nijmegen, The Netherlands

e-mail: N.Lubsen@science.ru.nl

\section{A. G. Purkiss - C. Slingsby}

Department of Biological Sciences, Crystallography,

Institute of Structural and Molecular Biology, Birkbeck College,

Malet Street, London WC1E 7HX, UK 


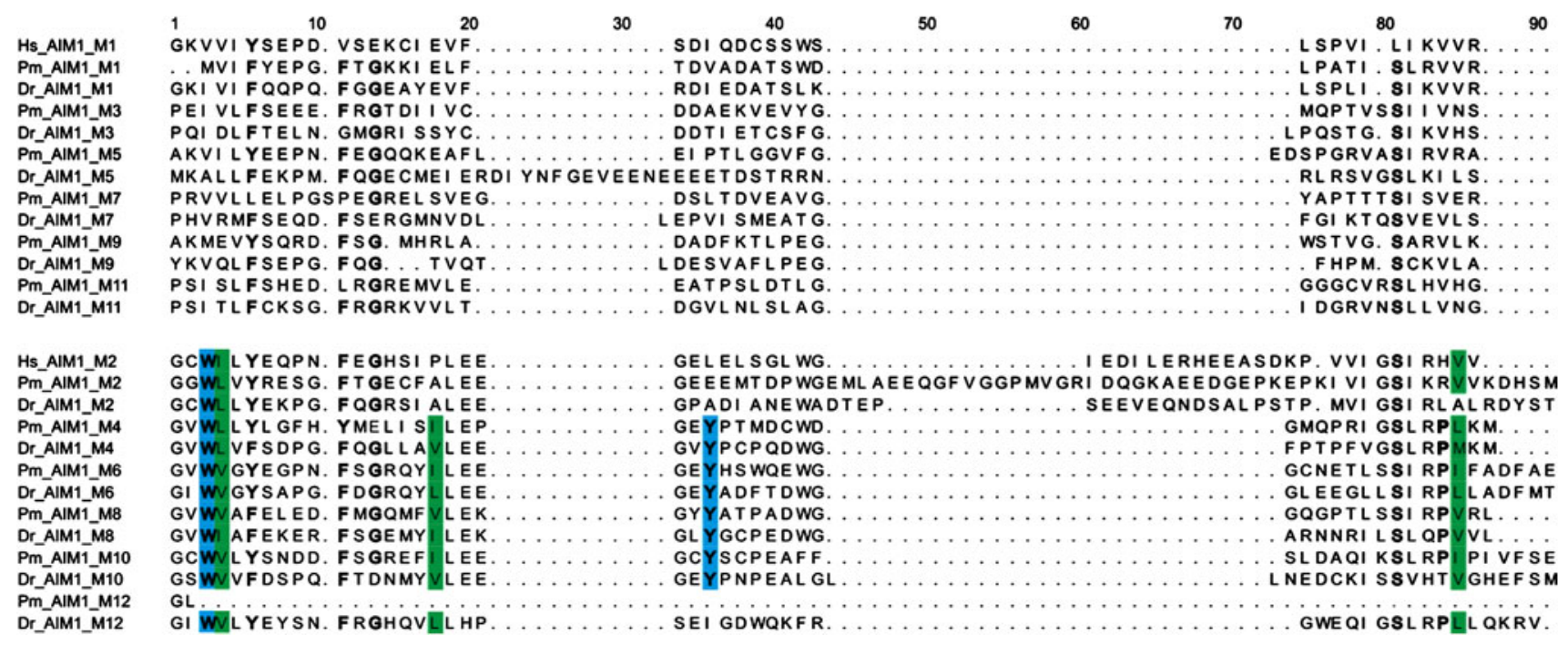

Fig. 2 Alignment of $P$. marinus AIM1 $\beta \gamma$-crystallin motifs. The AIM1 $\beta \gamma$-crystallin motifs found in the $P$. marinus $(\mathrm{Pm})$ genome assembly are aligned with those of the $D$. rerio (Dr) AIM1 protein and with the first two $\beta \gamma$-crystallin motifs of human (Hs) AIM1. The alignment is split in the odd and even motifs. Structurally important residues are indicated as in Fig. 1

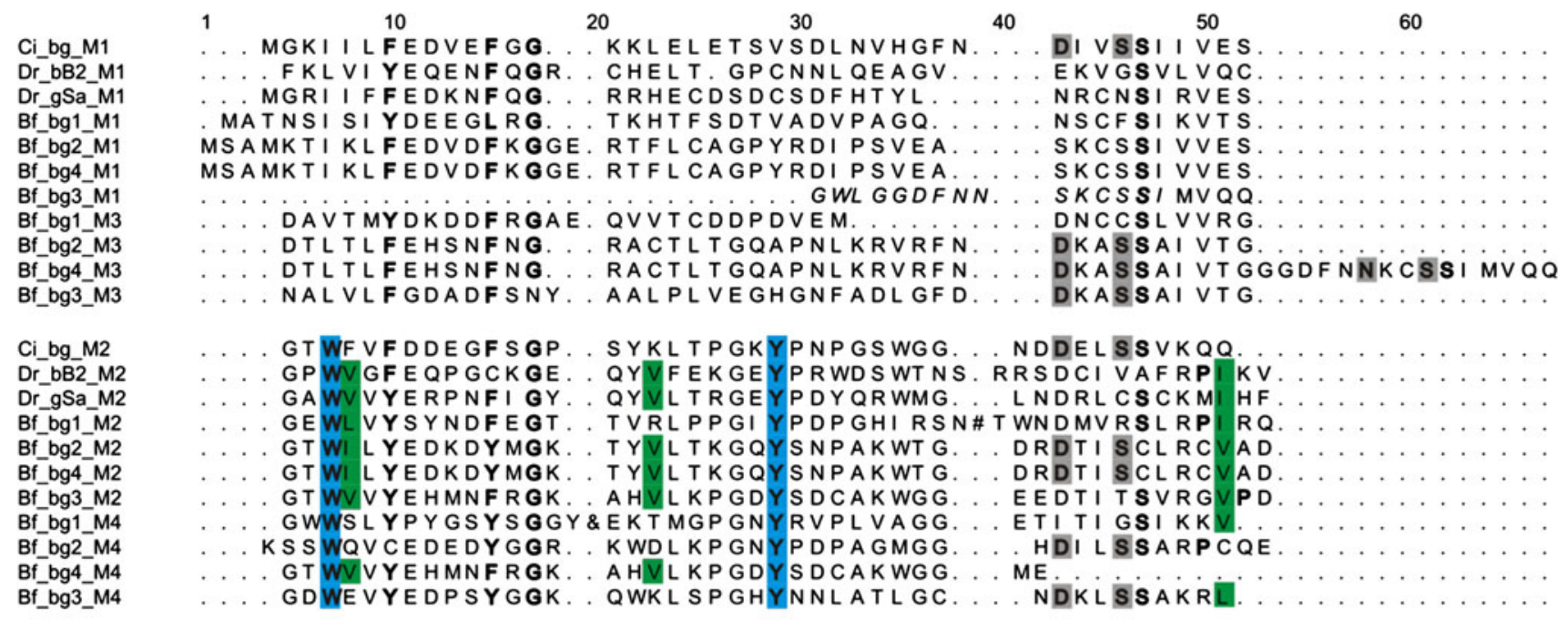

Bf_bg1_C-tail ECPYGPWPFPI DI PPLFPGTNVGLFHHVLPF

Bf_bg2_C-tail NPGI QLFEHADFKGQSVSCGHTGALVEG

Fig. 5 Alignment of $B$. floridae $\beta \gamma$-crystallin related sequences. The predicted protein sequences of $B$. floridae Bf-bg1, -bg2, -bg3 and -bg4 (acc.nr. FE565747, BW704196 and BW723025) are aligned with the $C$. intestinalis $\beta \gamma$-crystallin $\left(\mathrm{Ci} \_b g\right)$ and the $D$. rerio $\beta \mathrm{B} 2-\left(\mathrm{Dr} \_\mathrm{bB} 2\right)$ and $\gamma \mathrm{Sa}$-crystallin (Dr_gSa). The motifs are indicated as $M$ followed by the number. Structurally important residues are indicated as in
Fig. 1; calcium-binding residues are in grey. The sequence in italics in Bf-bg3 M1 was derived from the genomic sequence; the C-terminal extensions of Bf-bg1 and Bf-bg2 are shown below the alignment. The \# indicates the position of the HVNPANT insert in Bf-bg1 M2 and \& that of PPSNM in Bf-bg1 M4. The alignment is split in the odd and even motifs 